\title{
OPTIMISATION OF A TWO-WIRE THERMAL SENSOR FOR FLOW AND SOUND MEASUREMENTS
}

\author{
J.W. van Honschoten, G.J.M. Krijnen, V.B. Svetovoy, H-E. de Bree, M.C. Elwenspoek \\ Transducers Technology Laboratory, MESA+ Research Institute, University of Twente
}

The Netherlands

\section{ABSTRACT}

The Microflown is an acoustic sensor measuring particle velocity instead of pressure, which is usually measured by conventional microphones $[2,3,4]$. In this paper an analytical model is presented to describe the physical processes that govern the behaviour of the sensor and determine its sensitivity. The Microflown consists of two heaters that act simultaneously as sensors. Forced convection by an acoustic wave leads to a small perturbation of this temperature profile, resulting in a temperature difference between the two sensors. This temperature difference, to which the sensitivity is proportional, is calculated with perturbation theory. Consequently the frequency dependent behaviour of the sensitivity is analysed; it is found that there are two important corner frequencies, the first related to the time constant velocity of heat diffusion between the sensors, the second related to the heat capacity of the heaters. The developed model is verified by experiments.

In [12] a very good model is given for the performance of the Microflown in a channel, i.e. with both heaters between fixed walls walls in the positive and negative z-direction. Here, a model is presented that describes the situation of the present used sensors: without walls under and above them. Model predictions are compared to experimental results.

\section{INTRODUCTION}

The Microflown is an acoustic sensor based on a thermal principle [1]. Since its invention in 1994, [2] it is mostly used for measurement purposes (1D and 3D-sound intensity measurement [3], [4], [5], [6] or acoustic impedance [7], [8] and pressure [9]) Nowadays the Microflown is also used as an add-on microphone for professional recording purposes [14]. This is because the Microflown is, contrary to pressure gradient microphones, very sensitive to low frequency sound waves. The Microflown can also be used for measuring DC-flows [10]. Sound-energy determination and threedimensional impulse response measurement are under investigation.

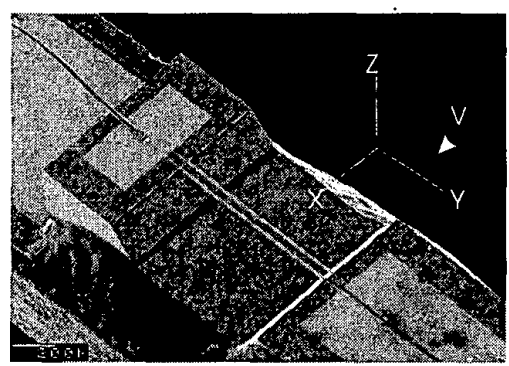

Fig. 1: SEM Photo of a part of a bridge type Microflown. At the top of the sample a wire-bond is visible. The sample is glued on a printed circuit board, the glue can be seen at the side of the sample.

The Microflown used in this work consists of two very closely spaced bridges (spacing $350 \mu \mathrm{m}$ ) of silicon nitride with a platinum electrode pattern on top of them. This is a two-sensor type ('TSM'), the other possible configuration is the 'SHS'(sensor-heater-sensor). A SEM photograph of a Microflown is depicted in Fig. 1.
Dimensions of the two wires (bridges) are $1000 \times 10 \times 0.5 \mu \mathrm{m}(1 \times w \times h)$. The metal pattern is used as temperature sensor and as heater. The silicon nitride layers are mechanical carriers for the platinum resistor patterns. These resistors are heated to an operational temperature of ca. 200 to $400^{\circ} \mathrm{C}$ by an electrical current dissipating an electrical power $P_{e l}$. The resistance of the sensors increases linearly with their temperature. When particle velocity is present, it alters the temperature distribution around the resistors. The temperature difference of the wires is proportional to the particle velocity. Due to the particle velocity, the upstream sensor drops more in temperature than the downstream sensor. Because of the thermal mass of the sensors and diffusion effects, sensors cannot follow the thermal signal fully for increasing frequencies and the Microflown therefore exhibits a high frequency "roll off".

\section{MODELLING THE TEMPERATURE PROFILE}

In the following, a model is developed for a Microflown of two infinitely long heaters of width $2 L$ and negligible height, separated by a distance $2 a$ with $a$ much larger than $L$. These heated wires cause a temperature distribution of the air around them. This undisturbed temperature profile, not yet influenced by an acoustical wave, is calculated, and used as a starting point for calculation of the temperature difference of the heaters when a flow is applied, by adding a perturbation to the original temperature profile.

\subsection{THE STATIONARY SITUATION}

In [12], it is shown that the distribution of the temperature in the situation in which the fluid (i.c. the air) is in rest is shown to be a good starting point to describe the temperature change due to a sound wave by perturbation analysis. From the temperature difference between the sensors/heaters the sensitivity of the Microflown is deduced. In [12] both unperturbed and perturbed temperature profiles are written as a Fourier series, an expansion in harmonics.

Here, it is aimed to describe the temperature for the configuration without walls, i.e. 'heat sinks', in the z-direction (under and above the heaters), and to find an analytical expression, not only a series, for the temperature difference and so for the sensitivity.

The stationary heat transport equation for this geometry yields

$-\nabla(k \nabla T)=Q$

where $k=k(T)$ represents the heat conductivity of the air, and $Q$ the heat production per unit of time and volume. Suppose a heater of infinite length in the $\mathrm{y}$-direction $\left(l_{\mathrm{y}}\right.$ much larger than other dimensions), of width $2 L$ in the $x$-direction and negligible height in the z-direction. Since the power per unit length is $P_{e /} / l_{y}$ for $Q$ can be written:

$Q=\frac{P}{2 l_{y} L} F(x) \delta(z)$

where $F(x)=0$ for $|x|>L ; F(x)=1$ for $|x|<L$. Neglecting the temperature dependence of $k$ (see [12]), the differential equation for $T$ reads:

$$
\left(\partial_{x}^{2}+\partial_{z}^{2}\right) T=-\frac{P}{2 l_{y} k L} F(x) \delta(z)
$$


Since the length $l_{y}$ is taken large and the temperature along this direction is assumed to be constant, the problem is reduced to two dirnensions, contrary to [12]. To solve (3), the analogue with electrostatic theory is used: an infinitely long wire of uniform charge density. In two dimensions the electrostatic potential behaves as $\ln (r)$, having singularities at both $r \rightarrow 0$ and $r \rightarrow \infty$. Of course the solution for $T$ should go to a finite temperature. Physically, the problem is actually three-dimensional; the two-dimensional approximation only yields for distances much smaller than $l_{y}$. The boundary condition for $T$ has to be fulfilled at about $r \sim l_{y}$.

Considering a very narrow, infinitely long heater, the differential equation for the temperature becomes:

$\left(\partial_{x}^{2}+\partial_{z}^{2}\right) T=-\frac{P}{l_{y} k} \delta(x) \delta(z)$

Using the electrostatic analogue, the solution of Eq. (4) is found as:

$T(x, z)=A \ln \left(\sqrt{x^{2}+z^{2}}\right)$ with $A=\frac{-P}{2 \pi k l_{y}}$

The argument of In has dimension 'meters' and should be made dimensionless. With $l_{y}$ of the proper dimension meters, one can write

$T(x, z)=\frac{-P}{2 \pi k l_{\cdot}} \ln \left(\frac{r}{l_{y}}\right)$

Only some constant should be added. To avoid the, unrealistic, singularity at the origin, a finite heaters width is considered. Many infinitely thin and long wires are placed in the heater and subsequently the average value of the potential is taken to obtain

$T(x, z)=\frac{-P}{2 \pi k l_{y}} \frac{1}{2 L} \int_{-L}^{L} \ln \frac{\sqrt{\left(x-x^{\prime}\right)^{2}+z^{2}}}{l_{y}} d x^{\prime}$

Evaluating the temperature in $(x, z)=(0,0)$ yields

$$
T(0,0)=\frac{-P}{2 \pi k l_{y}}\left(\ln \left(L / l_{y}\right)-1\right)
$$

and the temperature is zero far from the heater at a distance $\sim l_{y}$. This temperature distribution is taken as a starting-point to proceed and to consider the influence of the sound wave. However, Eq. (7) is only an approximation, valid for $L<<l_{y}$.

It may be obvious that during the calculation of the temperature profile above, only one heater was considered, see Eq.(3). The situation of two heaters of width $2 L$ and mutual distance $2 a$ is no fundamentally different, because of the linearity of the heat equation. The latter configuration can therefore be solved from only one heater. See also [12].

\subsection{INFLUENCE OF AN ACOUSTICAL WAVE ON THE TEMPERATURE PROFILE}

Now the change of the temperature profile due to the acoustical wave, described as a small perturbation, is calculated. The convection term involved is assumed to be small.

With this particle flow, the temperature distribution should obey:

- $\rho c_{p}\left(\partial_{t} T+\nu \nabla T\right)-\nabla(k \nabla T)=Q$

where $v=v e^{i \omega t}$ represents the (particle) flow velocity, $\rho$ the density and $c_{p}$ the specific heat capacity of the fluid. The convection term proportional to $v$ (only $v_{x}$ ) causes an asymmetry of the temperature distribution. Since $v$ is assumed to be small, Eq. 9 can be solved from Eq. 1 with a little addition $\delta T(t)=\delta T e^{i \omega t}$, proportional to $v$. So, in first order terms of $\delta T$ :

$\partial_{t} \delta T-D \nabla^{2} \delta T=-v \partial_{x} T$ with $D=k / \rho c_{p}$ the thermal diffusion coefficient of the air. In this approach the assumption is made that the heaters do not significantly influence the velocity profile of the flow, and that boundary layer effects are negligible.

Now Eq. (10) should be solved, using the boundary conditions $\delta T$ .If the perturbation $\delta T$ is taken as a harmonic signal, the new equation for $\delta T$ becomes

$i 2 \pi f \delta T-D\left(\partial_{x}^{2}+\partial_{z}^{2}\right) \delta T=-v_{x} \partial_{x} T$

After substitution of $\partial_{x} T$ from Eq.(7), and elimination of the $z$ coordinate by defining the Fourier transforms $\delta \bar{T}(x, q)$ and $\bar{T}(x, q)$, a mathematical deduction leads to a solution for $\overline{\delta T}(x, q)$ :

$\delta \bar{T}=i \frac{v}{2 \pi f} \frac{P}{4 \pi k l_{y}}\left(\frac{\sinh (|q| L)}{|q| L} e^{-|q| x}-\frac{\sinh (K L)}{K L} e^{-K x}\right)$

The solution is written for the range $x>L$, since this is the interval of interest for $\delta T$. When the situation of two heaters (sensors) is considered, the function for $T$ consists of two terms of the form (7), e.g. $T_{\text {left herter }}$ and $T_{\text {right. }}$, each term shifted over a certain distance $x$. Actually the differential equation for $\delta \bar{T}(x, q)$ should then be solved with both terms for $\partial_{x} T(x, q)$. In [12] it is shown that the sensitivity of the sensors in the TSM-configuration, is in fact similar to the sensitivity of the SHS-configuration, for which the analysis above applies, when the distance $a$ between heater and sensor in the SHSsituation equals the distance between heater and sensor in the TSMconstruction. Concluding, the expression for $\delta T(x, q)$ in Eq. (12) is sufficient to calculate the temperature difference for the two-sensorsconstruction, due to the flow.

Finding an analytical form of the Fourier transform of this expression is troublesome because of the first term between brackets, but the point of interest is $\delta T(x, z)$ at $z=0$. So, to know the correction to the sensor temperature $\delta T(x, 0)$, Eq. (12) is averaged over the sensor width (from $x=-L$ to $x=L$ ) and then follows

$\delta T(x, 0)=\frac{2 i v}{2 \pi f} \frac{P}{4 \pi k l_{y}} \int_{0}^{\infty}\left(\left[\frac{\sinh (q L)}{q L}\right]^{2} e^{-q x}-\left[\frac{\sinh (K L)}{K L}\right]^{2} e^{-K x}\right) d q$

Subsequently, the sensors' temperature difference is calculated from

$\Delta T=2 \delta T(a, 0)$

The integral in Eq. (13) can only be evaluated analytically if the approximation of $L \rightarrow O$ is made (so $L<<a$ ). The squared terms in front of the exponents then go to $l$, and using some integration theory, one obtains for $\Delta T$

$\Delta T=\frac{v P a}{\pi k l_{y} D \bar{f}}\left(1-\sqrt{i \bar{f}} K_{1}(\sqrt{i \bar{f}})\right)$

with $K_{l}(x)$ the modified Bessel function of the first kind, and the dimensionless frequency $\bar{f}=f 2 \pi a^{2} D^{-1}$. This corresponds to $\bar{f}=f / f_{D}$, with $f_{D} \equiv D / 2 \pi a^{2}$ the thermal diffusion frequency, an important scale factor in the expression (15). For large $\bar{f}$, the real part of $\Delta T$, the part of interest, behaves as $f^{-1}$. For $\bar{f} \ll 1$, so $f \ll f_{D}$, the behaviour of the Bessel function is as

$\sqrt{i \bar{f}} K_{1}(\sqrt{i \bar{f}})=1+\frac{i \bar{f}}{2} \ln \frac{\sqrt{i \bar{f}}}{2}+O(\bar{f})$.

of which the real part is of importance. So, for $f$ small compared to $f_{D}$, $\Delta T$ does not depend on frequency. Eq.(15) also shows that $\Delta T$ is 
proportional to the dissipated power per unit length $P / l_{y}$, as well as to the velocity $v$. From $\Delta T$, the sensitivity $S$ is easily calculated. This sensitivity $S$ is proportional to $\Delta T$ and therefore a function of frequency and distance $a$ between the sensors. The form

$$
\Delta T(a, f)=\operatorname{Re}\left(\operatorname{const} \frac{1}{f}\left(\frac{1}{a}-\sqrt{i f \frac{2 \pi}{D}} K_{1}\left(a \sqrt{i f \frac{2 \pi}{D}}\right)\right)\right)
$$

is plotted in Fig.2a as a function of $a$ for different frequencies, where $\Delta T(a, f)$ is logarithmically plotted as a function of $f$, with $a$ as parameter, in Fig.2b.

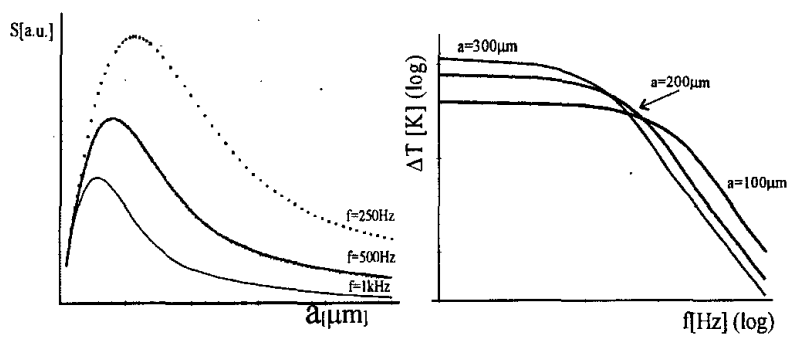

Fig. 2a: Sensitivity $S(a, f)$ for $f=250 \mathrm{~Hz}$ (points), $500 \mathrm{~Hz}$ (dotted) and lkHz. Fig. 2b: Output signal $\Delta T(a, f)$, on a logarithmic scale, for $a=100 \mu \mathrm{m}, a=200 \mu \mathrm{m}$ and $a=300 \mu \mathrm{m}$.

\subsection{THE EFFECT OF THE HEAT CAPACITY OF THE SENSORS}

The next step in the approach is taking the heat capacity of the sensors into consideration. In Eq. 9 then an additional term appears:

$$
\begin{aligned}
& \left(\rho c_{p}\right)_{a i r}\left(\partial_{t} T+\vec{v} \nabla T\right)-h\left(\rho c_{p}\right)_{P t}\left(F(x-a) \partial_{t} \delta T+F(x+a) \partial_{t} \delta T\right) \\
& -\nabla(k \nabla T)=Q
\end{aligned}
$$

If, again, a small perturbation $\delta T$ is added to this equation, the same approach as to Eq.(10) leads to $\left(\left(\rho c_{p}\right)_{P_{t}}\right.$ represents the density times the heat capacity of the (platinum) sensor), Eq. (19):

$$
\partial_{t} \delta T-D \nabla^{2} \delta T=-v \partial_{x} T+h \frac{\left(\rho c_{p}\right)_{P t}}{\left(\rho c_{p}\right)_{\text {air }}}\left(F(x-a) \partial_{t} \delta T+F(x+a) \partial_{t} \delta T\right)
$$

assuming the two beams of the two-sensor-Microflown at $x= \pm a$. Analogously, $\delta T(x, z)$ is transformed into $\delta \bar{T}(x, q)$, and for the limit of narrow beams, $F(x)$ is approximated by $F(x)=2 L \delta(x)$.

It is not easy to solve the resulting equation, since it relates $\overline{\delta T}(x, q)$ and its derivatives to the value of $\overline{\delta T}(x, q)$ in $x=a$. But since the point of interest of the solution $\delta \bar{T}(x, q)$ is only $\delta \bar{T}(a, q)$, an expression for $\delta \bar{T}(a, q)$ and therefore for $\delta T(a, z)$ in $z=0$ can be found, by substituting $x=a$ in the equation. Then $\Delta T$ obeys Eq. 20:

$$
\Delta T=2 \delta T(a, 0)=\operatorname{Re}\left\{\left(\frac{1}{1+2 i \beta \frac{2 \pi f}{D}}\right) \frac{2 \nu P}{4 \pi^{2} k l_{y} f}\left(\frac{1}{a}-\sqrt{i f \frac{2 \pi}{D}} K_{1}\left(a \sqrt{i f \frac{2 \pi}{D}}\right)\right)\right\}
$$

with $\beta=L h \frac{\left(\rho c_{p}\right)_{P t}}{\left(\rho c_{p}\right)_{a i r}}$. Comparing Eq.(20) to the former expression for the temperature difference, Eq. (15), it is noticed that because of the heat capacity of the heaters, a factor $1 /\left(1+2 i \beta \frac{2 \pi f}{D}\right)$ appears in front. In terms of frequency dependent transfer functions, this factor corresponds to a first order transfer function with corner frequency

$$
f_{h c}=\frac{D}{4 \pi \beta}=\frac{D}{4 \pi L h} \frac{\left(\rho c_{p}\right)_{a i r}}{\left(\rho c_{p}\right)_{p t}}
$$

Thus, this first factor of Eq.(20) corresponds to a first order transition with a corner frequency determined by the heat capacity of the sensors

\subsection{BEHAVIOUR OF THE SENSITIVITY FUNCTION}

The behaviour of (15) is similar, but not equal to that of a first order transfer function, with a corner frequency directly related to $f_{D}$ (see Fig.2b). Nevertheless, there is a large region around the thermal diffusion frequency $f_{D}$, for which the function slowly decreases (on a logarithmic scale): although the thermal frequency is an important scale factor in the expression for $\Delta T$, it does not equal the corner frequency of the first order transition. The sensitivity for steady ('DC')flow is found from Eq.(20), taking $\lim (f \downarrow 0)$. Then the low frequency sensitivity $S(0)$ is calculated as

$$
S(0)=\frac{v_{x} P a}{8 D k l_{y}}
$$

If, however, the behaviour of the function for large $f$, is compared to that of a first order transfer function $H(f)=S(0) / \sqrt{1+\left(f / f_{d}\right)^{2}}$ with corner frequency $f_{d}$, then an apparent corner frequency of function (15) could be calculated. This apparent first order corner frequency, defined as the intersection of the two asymptotes, is then

$f_{d}=\frac{8}{\pi} f_{D}=\frac{4 D}{\pi^{2} a^{2}}$

Assuming $T=600 \mathrm{~K}, \quad a=300 \mu \mathrm{m}, D=7.7 \cdot 10^{-5} \mathrm{~m}^{2} \mathrm{~s}^{-1}$, yields a thermal diffusion frequency $f_{D}=D / 2 \pi a^{2}=136 \mathrm{~Hz}$, and $f_{d}=\frac{8}{\pi} f_{D}=350 \mathrm{~Hz}$.

Both the corner frequency $f_{h c}$, frequency $f_{d}$ and $S(0)$ depend on the thermal diffusion coefficient, which is strongly temperature dependent; $D$ increases from about $1.59 \cdot 10^{-5} \mathrm{~m}^{2} \mathrm{~s}^{-1}$ at $T=250 \mathrm{~K}$ to ca. $7.69 \cdot 10^{-5} \mathrm{~m}^{2} \mathrm{~s}^{-1}$ at $T=600 \mathrm{~K}$.

\section{COMPARISON TO EXPERIMENTS}

Microflowns of varying distance $a$, width $2 L$, and length $y$ have been designed. After the mask design, the technology consisted of deposition and patterning of $\mathrm{Si}_{3} \mathrm{~N}_{4}$, deposition of a $100 \mathrm{~nm}$ metal $(\mathrm{Cr} / \mathrm{Pt})$ layer and after photolithography, (KOH-)etching of $\mathrm{Cr} / \mathrm{Pt}$, and $\mathrm{Si}_{3} \mathrm{~N}_{4}[1]$. To analyse experimentally the sensitivity of these Microflowns, measurements in a standing wave tube were performed. Inside a long tube with at one side a sound source generating a sine wave, and at the other side a reflecting cross section with a reference microphone in it, standing wave patterns occur. Somewhere along this standing wave tube a Microflown is placed, so that from the ratio between the signals of reference microphone and Microflown the sensitivity of the latter can be derived ([1],[11]). In the experiments the sensitivity was measured as a function of frequency for frequencies $0<f<4 k H z$, using Microflowns of varying distance $a$ between the heaters and varying width $L$ and length $l_{y}$ of the heaters.

For an investigation of the mutual variance of sensors of the same geometry, and the errors due to the measurement set-up, at first several sensors of exactly the same geometry were successively measured. From these results it could be concluded that the reproducibility of the measurements was better than $1 \%$.

Then $L, a$ and $l_{y}$ were successively varied. It was verified that varying $l_{y}$ did not influence the sensitivity significantly, corresponding with theory. The parameter $a$ was given values between 100 and $500 \mu \mathrm{m}$, and the frequency dependent transfer function of the Microflown with respect to the reference microphone was successively measured. These results were compared to the theoretically expected functions, for both amplitude and phase of $S(f)$. (See Fig.3, 4). The proportionality (scale) factor between $S$ and $\Delta T$, for fitting, was normalised once to the average of all measurements of $S(0)$. From these functions it could be checked if both dependences on parameter $a$, the low-frequency amplitude $S(0)$ and the characteristic (thermal) frequency, corresponded with theory. The influence of the heat capacity of the heaters, determining the second corner frequency $f_{h c}$, was investigated by altering the width $L$ of the sensors. Again the various measurements were fitted by theoretical curves. As 
theoretically expected, the corner frequency $f_{h c}$ is proportional to the width $L$.

It is seen that the model appears to describe rather accurately the frequency dependence of the sensor. This is also illustrated by the dependence of $S_{(=2000 \mathrm{~Hz})}$ on $L$ (Fig.5), which is a measure of the corner frequency. Experiments show also proportionality of sensitivity $S(0)$ to $a$ for small $a(a<\mathrm{ca} .200 \mu \mathrm{m})$ and $S(0)$ obeys Ei.(22). For larger $a$ however, $S(0)$ decreases with the mutual distance. Therefore the deduced model appears to describe accurately the frequency dependent behaviour and the values of the characteristic frequencies, and predicts reasonable values of $S(0)$ for $a$ not too large. For distances $a$ of the order of $l_{y}$ and larger it cannot describe yet the low-frequency behaviour. This aspect is currently being investigated.

\section{SUMMARY AND CONCLUSIONS}

In the previous paragraphs a model is presented that predicts the temperature difference $\Delta T$ as result of a particle velocity of amplitude $v$. The model is valid as long as the wires of the Microflown can be considered long and thin, compared to the mutual distances. A certain particle velocity $v$ is assumed, and since $v$ is relatively small, perturbation theory is justified to calculate $\Delta T$ and thus the (frequency dependent) sensitivity. For the investigated geometries, length $l_{y}$ of the heaters ca. $1-1.5 \mathrm{~mm}$, mutual distances $a$ between 100 and $500 \mu \mathrm{m}$, and widths $L$ of $1-5 \mu \mathrm{m}$, the theory is confirmed by experiments in the whole frequency range $10-4500 \mathrm{~Hz}$. For mutual distances $a$ larger than ca. $200 \mu \mathrm{m}$, the frequency behaviour of the sensitivity corresponds to theory as well, but the model does not describe yet the value of the low-frequency sessitivity. This value $S(0)$ for $a$ of magnitudes $-l_{y}$, is therefore a point of further research.

\section{REFERENCES}

[1] H-E. de Bree et al., The Microflown: a novel device measuring acoustical flows, Sensors and Actuators A 54 (1996) $552-557$

[2] H-E. de Bree, Lammerink, Elwenspoek, Fluitman, Patent PCT/NL95/00220, Use of a fluid flow measuring device as a microphone and system comprising such a microphone.

[3] W.F. Druyvesteyn et al, A new sound intensity probe; comparison to the Bruel \& Kjaer p-p probe, 104th AES Convention Amsterdam; pre-print 4651, 1998

[4] W.F. Druyvesteyn et al., A New acoustic measurement probe; the Microflown, IOA London, 1999.

[5] H-E. de Bree et al., Three-dimensional sound intensity measurements using microflown particle velocity sensors, MEMS '99, Orlando.

[6] H-E de Bree et al., Realisation and calibration of a novel half inch p-u sound intensity probe, $106^{\text {th }}$ AES Munchen,' 99

[7] H. Schurer et al., Comparison of two methods for measurement of horn input impedance, Proceedings of the 100th AES convention, Copenhagen, 1996.

[8] F.J.M. van der Eerden et al., Experiments with a new acoustic particle velocity sensor in an impedance tube Sensors and Actuators A69, (1998) 126-133.

[9]. H.E. de Bree et al., A method to measure apparent acoustic pressure, flow gradient and acoustic intensity using two micromachined flow microphones, Eurosensors $\mathrm{X}$, Leuven, 96

[10] H-E. de Bree et al., Bi-directional fast flow sensor with a large dynamic range, MME, 1998

[11] Intensity measurements in various rooms / a new intensity probe (W.F. Druyvesteyn et al, 16th ICA and 135th ASA meeting; Seattle June 1998, Vol. 4, pp. 2827-2828.)

[12] 'Model of the $\mu$-flown microphone', V.B. Svetovoy, I.A.Winter, Sensors \&Actuators 86 (2000), 171-181.

[13] Advanced Calculus, M.R.Spiegel, 1974.

[14] H.E. de Bree, Add-on Microflown for a high-end pressure gradient microphone, AES, Los Angeles 2000.
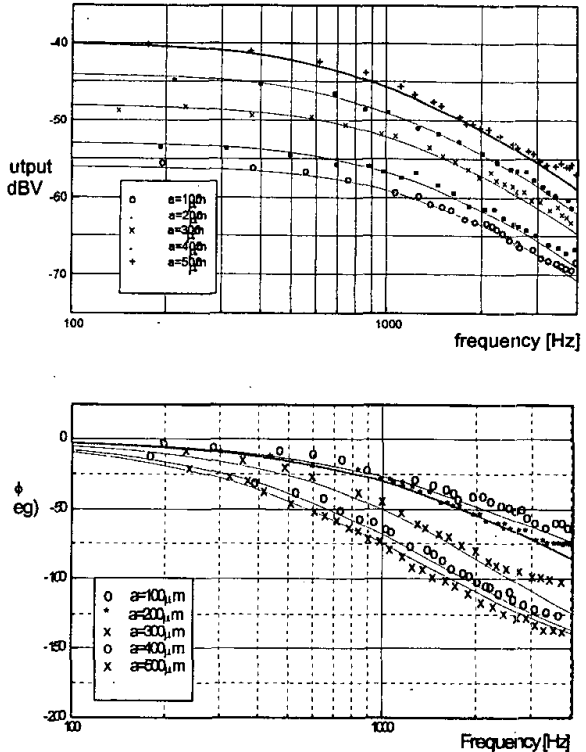

Fig.3 Amplitude(upper) and phase(lower) of the sensitivity as a function of $f$, for varying $a$. Curves represent model calculations.

aput

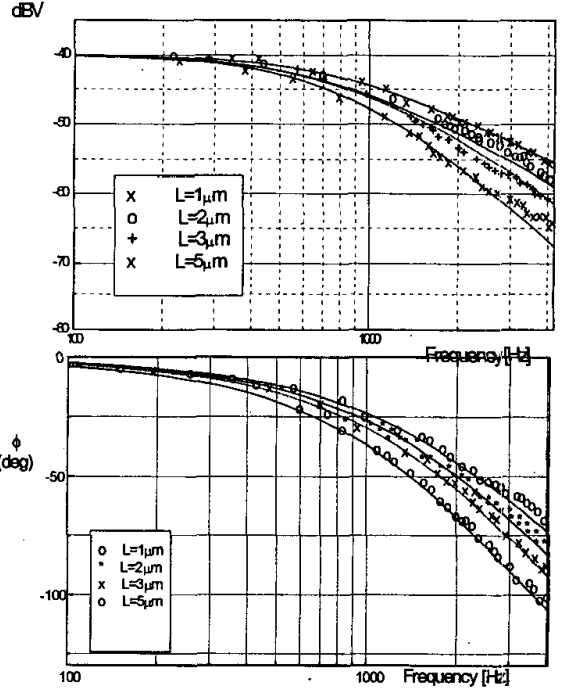

Fig.4 Amplitude(upper) and phase (lower) of the sensitivity as a function of $f$, for varying width $L$ of the sensors. Curves represent calculated values.

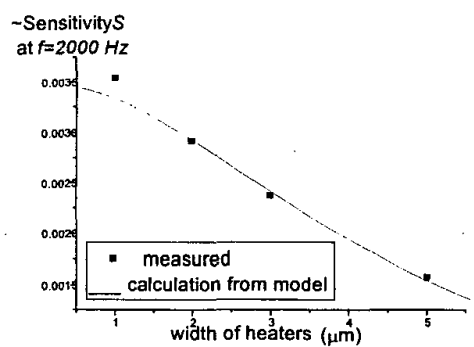

Fig.5:Calculated and measured sensitivity at $f=2000 \mathrm{~Hz}$, for varying width $L$ of the sensors. 